\title{
Synaptic Functions in Rat Sympathetic Neurons in Microcultures. III. A Purinergic Effect on Cardiac Myocytes
}

\author{
E. J. Furshpan, D. D. Potter, and S. G. Matsumoto \\ Department of Neurobiology, Harvard Medical School, Boston, Massachusetts 02115
}

In the first two of this series of papers, a sensitive microculture procedure was used to show that rat sympathetic neurons grown singly on small islands of heart cells release norepinephrine (NE) and/or acetylcholine (ACh). We report here the release of a third transmitter in response to stimulation of these neurons. This agent was recognized by its effect on the cocultured cardiac myocytes: an inhibition of beating or a hyperpolarization that, in contrast to cholinergic inhibition, was unaffected by atropine (up to $5 \mu \mathrm{M}$ ). Evidence described here indicates that this agent was primarily adenosine (or a closely related compound): the atropine-resistant myocyte inhibition was antagonized by adenosine-receptor blockers [8-phenyltheophylline, theophylline, 7-(2-chloroethyl) theophylline] and was attenuated by an enzyme (adenosine deaminase) that hydrolyzes adenosine to pharmacologically inactive inosine. Many of the neurons, whether initially dissociated from ganglia of newborn or adult rats, evoked this purinergic response, almost always in combination with adrenergic and cholinergic responses. In a few cases it was the only detectable response. The relative strength of the adrenergic, cholinergic, and purinergic responses varied widely from neuron to neuron, suggesting that the adrenergic and purinergic or the cholinergic and purinergic agents were not stored at constant stoichiometric ratios.

In the preceding paper (Potter et al., 1986), we described the gradual transition from adrenergic to cholinergic function, as well as the simultaneous expression of the two functions, in individual sympathetic neurons grown in microcultures with cardiac myocytes. In the course of these experiments we sometimes observed neuronally evoked hyperpolarizations of the myocytes that were reduced but not abolished by atropine (usually $0.1 \mu \mathrm{M})$. In a few of these cases the responses were eliminated by higher concentrations of atropine $(0.5-1 \mu \mathrm{M})$. In several cases the response amplitude was not further reduced when the atropine concentration was increased, but these puzzling observations were not pursued. Then, in a particular plating we encountered a high incidence of atropine-resistant myocyte hyperpolarizations (evoked by at least 13 of 18 neurons tested). Many of these neurons, for reasons we do not understand, failed to develop cholinergic function, even after 10-15 weeks in cul-

\footnotetext{
Received Dec. 12, 1984; revised Sept. 18, 1985; accepted Sept. 23, 1985.

Expert assistance was provided by Robert Bosler, Delores Cox, James LaFratta, Michael LaFratta, Joseph Gagliardi, Ibrahim Houri, Doreen McDowell, Geraldine Spencer, Shirley Wilson, and Vivienne Yee. A ${ }^{60} \mathrm{Co}$ source was kindly made available by Drs. J. Epstein and J. D. Little. Drs. Linda Chun, Allison Doupe, Eve Wolinsky, and Geraldine Spencer provided NGF. We are indebted to many colleagues for helpful discussion, especially Drs. Patrick Hogan and Paul Patterson. This work was supported by NIH Grants NS03273, NS11576, NS02253, and a Grant-in-Aid from the American Heart Association (78-964).

Correspondence should be addressed to Professor E. J. Furshpan, Department of Neurobiology, Harvard Medical School, 25 Shattuck Street, Boston, MA 02115.

Copyright @ 1986 Society for Neuroscience $0270-6474 / 86 / 041099-09 \$ 02.00 / 0$
}

ture in the presence of heart cells. In the absence of any cholinergic response, we observed neuron-evoked hyperpolarizations that were completely insensitive to atropine, and this initiated a study of the pharmacology of this effect. In this paper we present evidence that it is purinergic.

Purines have been of interest in the control of the cardiovascular system since Drury and Szent-Gyorgyi (1929) identified, in extracts of bovine heart, adenosine and adenine nucleotides as active agents causing vasodilation and cardioinhibition. These compounds have repeatedly been found to evoke inotropic and chronotropic inhibition of the heart in a number of species (see Burnstock, 1980, for a review); they are generally effective on the heart in the micromolar range and can be nearly as active as acetylcholine (Belardinelli and Isenberg, 1983; Hartzell, 1979), although different parts of the heart may differ in their responsiveness (e.g., Burnstock and Meghji, 1981, 1983; Schrader et al., 1979).

The effects of purines have been extensively investigated and are of interest in several contexts (see Baer and Drummomd, 1979). It has been proposed that adenosine released from metabolically stressed myocytes is a mediator of reactive hyperemia in the heart and that adenosine similarly regulates blood flow in the brain, adipose tissue, and other tissues (for reviews, see Berne et al., 1971, 1983). Adenosine and related compounds have effects, mostly inhibitory, on a variety of peripheral and central ncurons (sce Phillis and Wu, 1981; Phillis ct al., 1979; Ribiero, 1979). Evidence has recently been reported for adenosinergic transmission in the parasympathetic ganglion of the bladder of the cat (Akasu et al., 1984). The possibility that ATP serves as a transmitter in certain primary sensory neurons was raised by Holton and Holton (1954) and Holton (1959). In 1970 Burnstock et al. proposed that ATP or a related substance mediates nonadrenergic, noncholinergic inhibition in the gut; in 1971 Burnstock coined the useful term "purinergic" to refer to such transmission. Evidence for purinergic transmission has been reviewed extensively (e.g., Burnstock, 1979, 1980; Stone, 1981). Evidence has recently appeared for excitatory actions mediated by ATP and for co-release of ATP and NE in the vas deferens and bladder of guinea pig and cat (e.g., Fedan et al., 1982; Sneddon et al., 1982; Theobald, 1983; Westfall et al., 1983); see Muramatsu et al. (1981) and Sneddon and Burnstock (1985) for related studies on arteries.

Co-storage and co-release of ATP with catecholamines in the adrenal medulla has been much studied since the work of Hillarp and Thieme (1959), Blaschko et al. (1956), and Douglas and Poisner (1966a, b); see Van Dyke et al. (1977). ATP is present in purified fractions of cholinergic vesicles from elasmobranch electric organs (e.g., Wagner et al., 1978), and the release of ATP from nerve-muscle preparations following stimulation of motor nerves has been observed (e.g., Silinsky, 1975). Co-storage of much smaller amounts of ATP with NE in small and large 

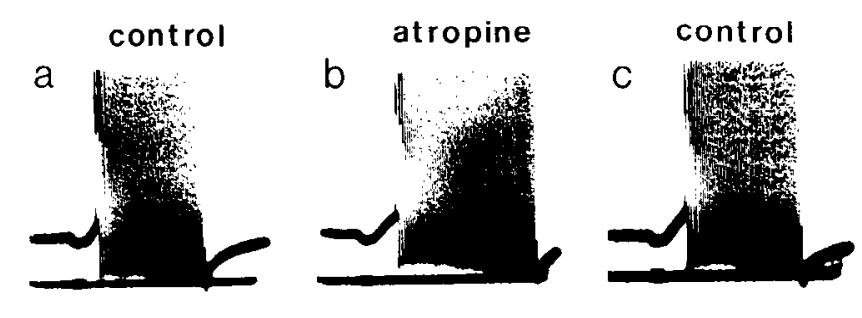

d aten/ptol

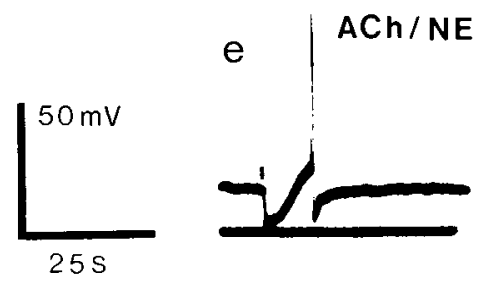

Figure 1. Atropine-resistant myocyte hyperpolarizations evoked by a solitary neuron $21 \mathrm{~d}$ in microculture. Upper traces, Intracellular recordings from a myocyte; lower traces, low-gain intracellular recordings from the neuron. $a-d$, The neuron was stimulated by brief trains of current pulses at $16 \mathrm{~Hz}$. Perfusion was with standard (control) solution in $a, c$, and $e$ and with standard solution containing $1 \mu \mathrm{M}$ atropine sulfate in $b$ or atenolol $(10 \mu \mathrm{M})$ and phentolamine $(0.1 \mu \mathrm{M})$ in $d$. $e$, Response to a brief puff of standard solution containing ACh (3 $\mu \mathrm{M})$ and $\mathrm{NE}(10 \mu \mathrm{M})$; time of application indicated by vertical bar. Note that the initial hyperpolarization in $a-d$ was unaffected either by atropine or by the atenolol-phentolamine mixture that blocked the delayed excitation. The voltage calibration applies to the upper traces in $a-e$; the myocyte impulses in $a-c$ are truncated.

granular vesicles of sympathetic neurons has been reported (e.g., Fried, 1980; Yen et al., 1976). It has long been recognized that secreted ATP may be broken down to the di- and monophosphates and to adenosine (as well as adenosine metabolites) by extracellular enzymes (e.g., Banks, 1966; Douglas and Poisner, 1966a; Paddle and Burnstock, 1974; Stevens et al. 1975).

Preliminary accounts of some aspects of this work have appeared (Furshpan et al., 1982; Potter et al., 1983).

\section{Materials and Methods}

The experiments reported here were made with microcultures of sympathetic neurons dissociated from the superior cervical ganglia of rats (both newborn and adult) and grown on islands of cardiac cells (myocytes and fibroblasts) dissociated from the hearts of newborn rats.

The methods for preparing and recording from the microcultures were identical to those described in the first paper of this series (Furshpan et al., 1986).

The adenosine-receptor antagonist 8-phenyltheophylline (8-PT) is not readily soluble in water. We prepared 8-PT solutions by either of two procedures: (1) Dry powder added to perfusion medium (Furshpan et al., 1986) at $1-10 \mu \mathrm{mol} / 1$ failed to dissolve completely; after several hours at $37^{\circ} \mathrm{C}$, with periodic agitation, the suspension was filtered (Nuclepore; pore diameter, $0.2 \mu \mathrm{m}$ ) and used. (2) 8-PT was dissolved at 3 mM in dimethylsulfoxide; an aliquot was diluted 1000 -fold with perfusion medium and filtered to remove any precipitate to make a solution that was nominally $3 \mu \mathrm{M} 8$-PT.

The stock adenosine deaminase preparation contained glycerol $(50 \%$ $\mathrm{v} / \mathrm{v}$ ) to stabilize the enzyme. Even after the enzyme was diluted (e.g., to $10 \mathrm{U} / \mathrm{ml}$ ), the glycerol concentration was appreciable. Appropriate concentrations of glycerol were added to the control solutions in a number of experiments. One unit $(1 \mathrm{U})$ of enzyme activity deaminates 1 $\mu \mathrm{mol}$ of adenosine $/ \mathrm{min}$ at $25^{\circ} \mathrm{C}$.

In some experiments, transient application of adenosine was made by ejection of brief "puffs" of drug solution from a micropipette (tip diameter, $5-10 \mu \mathrm{m}$ ) with brief pressure pulses controlled by a solenoid valve (see Choi and Fischbach, 1981). The pipette tip was usually positioned $25-50 \mu \mathrm{m}$ above the heart-cell island; the puff of ejected solution quickly spread over a large fraction of the island.

Drugs and their suppliers were as follows: acetylcholine chloride, adenosine, adenosine deaminase (type III), aminophylline, atropine sul- fate, norepinephrine bitartrate, theophylline: Sigma; 8-phenyltheophylline: Calbiochem; 7-(2-chloroethyl) theophylline: Aldrich; atenolol: Imperial Chemical Industries, Americas Inc. (Stuart); phentolamine (Regitine): Ciba-Geigy.

\section{Results}

In experiments described in the preceding papers (Furshpan et al., 1986; Potter et al., 1986), we found that some microcultured sympathetic neurons were detectably only adrenergic, some were detectably only cholinergic, while most exhibited adrenergic and cholinergic properties simultaneously. The subject of this paper is a third type of myocyte response evoked by stimulation of these sympathetic neurons: a noncholinergic, nonadrenergic inhibition. An example of this response is shown in Figure 1. A train of impulses in this solitary neuron evoked a myocyte hyperpolarization followed by a burst of beating $(a, c)$. This pattern of responses was reminiscent of that evoked by many adrenergic/cholinergic dual-function neurons (see Potter et al., 1986, Figs. $1 b_{1}$ and $2 b_{3}$ ). However, the hyperpolarization was unaffected by atropine at $1 \mu \mathrm{M}$ (Fig. $1 b$ ), $10 \times$ the concentration that abolished or substantially reduced the usual cholinergic inhibition (Furshpan et al., 1976, 1986). It was found in other experiments that the atropine-insensitive inhibition was also unaffected by the nicotinic antagonist hexamethonium (at $1 \mathrm{~mm}$ ). A $\beta$-adrenergic antagonist, atenolol, and an $\alpha$-adrenergic antagonist, phentolamine, blocked only the excitatory component of the response (Fig. 1d). Thus, it appeared that the hyperpolarization was not mediated by conventional cholinergic or adrenergic receptors. The myocytes on this island were sensitive to $\mathrm{ACh}$ as well as NE. Brief puffs of a solution containing $\mathrm{ACh}$ and NE evoked a substantial hyperpolarization followed by a brief excitation (Fig. 1e). Whatever the identity of the new agent, it was released with NE; this neuron displayed adrenergic/noncholinergic dual function.

Evidence that such atropine-resistant hyperpolarizations were not due to activation of any type of $\mathrm{ACh}$ receptor was obtained in an experiment on a microculture containing two neurons. Simultaneous recordings from the two neurons $\left(\mathrm{N}_{1}\right.$ and $\left.\mathrm{N}_{2}\right)$ are shown in Figure 2, $a$ and $b$. An action potential in $\mathrm{N}_{1}$, evoked directly by a current pulse, gave rise to an autaptic EPSP and an EPSP in $\mathrm{N}_{2}$, indicating that $\mathrm{N}_{1}$ was at least cholinergic (see Furshpan et al., 1986). A directly evoked action potential in $N_{2}$ did not give rise to an EPSP in either neuron. The effects of neuronal stimulation on the myocytes were then examined. Spontaneous beating of the myocytes was stopped by a train of impulses in either neuron ( $c$ and $d$; note difference in sweep speeds). However, the two inhibitions appeared to be mediated by different agents. As shown in $e$ and $f$, the inhibition evoked by $N_{1}$ was almost completely blocked by a mixture of receptor antagonists ( $3 \mathrm{~B}=$ "triple blocker") containing atropine, atenolol, and phentolamine, while the inhibition evoked by $\mathrm{N}_{2}$ was unaltered in 3B. $N_{1}$ and $N_{2}$ released their transmitters onto the same island of heart cells. The observations with $N_{1}$ indicated that these myocytes had cholinergic receptors that were predominantly or entirely muscarinic; if $\mathrm{N}_{2}$ had released $\mathrm{ACh}$ onto these myocytes, that component of the response should have been blocked by atropine. The simplest explanation for the difference in susceptibility to atropine of the two inhibitions is that $N_{1}$ and $N_{2}$ released different inhibitory transmitters. Atropinesensitive responses evoked by exogenously applied $\mathrm{ACh}$ provided additional evidence for the presence of conventional muscarinic receptors on these myocytes (e.g., Fig. 3, $b, d$, and $f$ ).

Observations described in the introduction raised the possibility that the atropine-insensitive inhibition was evoked by adenosine or an adenine nucleotide. The pharmacology of adenosine receptors has been extensively studied (e.g., Bruns, 1981; Smellie et al., 1979). Theophylline and certain related methylxanthines are useful blocking agents; 8-PT was about 25 -fold 

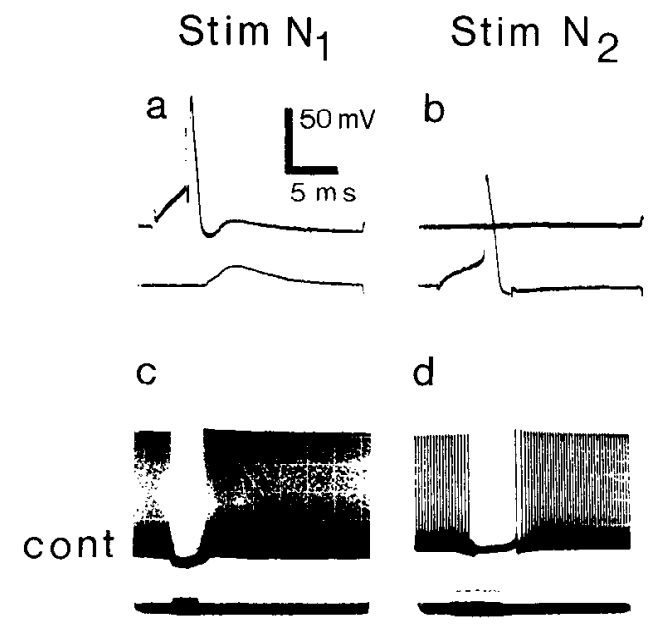

d
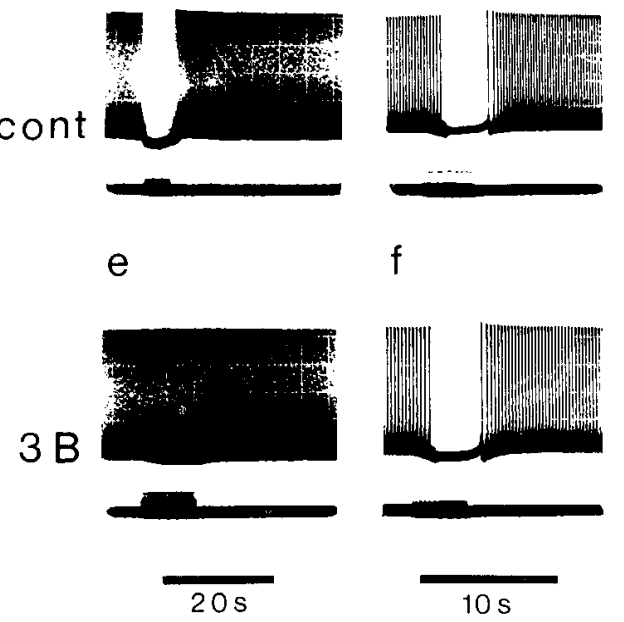

$10 \mathrm{~s}$

Figure 2. Comparison of the myocyte inhibition produced by two neurons $\left(\mathrm{N}_{1}\right.$ and $\left.\mathrm{N}_{2}\right)$ in the same microculture (for $92 \mathrm{~d}$ ). Suprathreshold current pulses were applied to $\mathrm{N}_{1}$ in $a, c$, and $e$ and to $\mathrm{N}_{2}$ in $b, d$, and $f$. Single pulses were applied in $a$ and $b$; trains of impulses at $8 \mathrm{~Hz}$ for 4-10 sec in $c-f$. Simultaneous recordings from $\mathrm{N}_{1}$ and $\mathrm{N}_{2}$ in $a$ and $b$ $\left(\mathrm{N}_{1}\right.$, upper traces; $\mathrm{N}_{2}$, lower traces); from $\mathrm{N}_{1}$ and a myocyte in $c$ and $e$ (myocyte, upper traces); and from $\mathrm{N}_{2}$ and the same myocyte in $d$ and $f$ (myocyte, upper traces). Perfusion was with control solution in $a-d$ and with $3 \mathrm{~B}$, a mixture of atropine sulfate $(1 \mu \mathrm{M})$, atenolol $(10 \mu \mathrm{M})$, and phentolamine $(0.1 \mu \mathrm{M})$, in $e$ and $f$. The voltage calibration $(50 \mathrm{mV})$ applies to $a$ and $b$ and the upper traces in $c-f$. Time calibrations: $5 \mathrm{msec}$ for $a$ and $b, 20 \mathrm{sec}$ for $c$ and $e, 10 \mathrm{sec}$ for $d$ and $f$. Note that $\mathrm{N}_{1}$ evoked cholinergic EPSPs, while $\mathrm{N}_{2}$ did not and that $3 \mathrm{~B}$ blocked the myocyte inhibition produced by $N_{1}$ but not that produced by $N_{2}$.

more effective than theophylline in one well-studied system, while 7-(2-chloroethyl) theophylline (7-CET) was about 5-fold more effective (Bruns, 1981). The pharmacology of adenine nucleotides is less well developed (see, however, Fedan et al., 1981; Sneddon and Burnstock, 1984).

\section{Effects of theophylline derivatives}

Figure 3 shows a test of the effect of 8-PT on the inhibition of the cardiac myocytes produced by neuron $\mathrm{N}_{2}$ of Figure 2 . The records were taken later in the experiment, when the myocytes no longer beat spontaneously. The left-hand columns $(a, c, e$, $g$, and $i$ ) show myocyte responses (upper traces) to a train of action potentials in neuron $\mathrm{N}_{2}$ (lower traces). The right-hand columns $(h, d, f, h$, and $j$ ) show myocyte responses to brief puffs of ACh (see Materials and Methods). The concentration of ACh in the puffer pipette was $3 \mu \mathrm{M}$. The responses to ACh mimicked rather closely the neuronally evoked responses $(a$ and $b$ ). However, when the mixture of blocking agents, 3B, that contained $1 \mu \mathrm{M}$ atropine was applied, the effect of $\mathrm{ACh}$ was abolished (d), while the neuron-evoked response was not appreciably affected (c). (The origin of the small depolarization caused by the $\mathrm{ACh}$ puff $(d)$ was not examined but was probably a mechanical artifact.) Washout of 3B restored the effect of $\mathrm{ACh}(f)$. The action
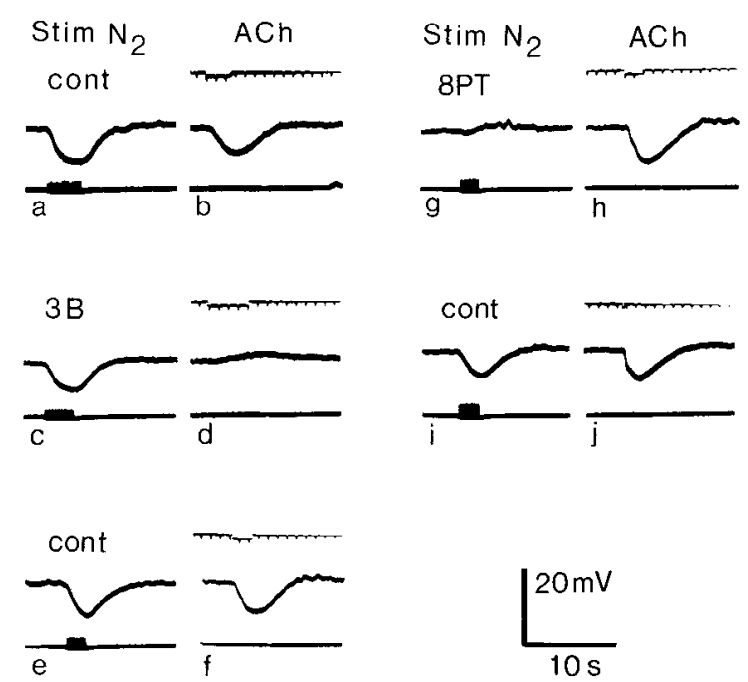

Figure 3. Pharmacological tests on myocyte responses evoked by neuronal stimulation or by puffs of $\mathrm{ACh}$; the same two-neuron microculture as in Figure 2 (neurons $92 \mathrm{~d}$ in vitro). Records $a, c, e, g$, and $i$ (lefthand columns) are myocyte responses (upper traces) to stimulation of the apparently noncholinergic neuron $\left(\mathrm{N}_{2}\right)$ at $8 \mathrm{~Hz}$; neuronal action potentials monitored on lower traces. Records $b, d, f, h$, and $j$ (righthand columns) are myocyte responses (middle traces) to puffs of ACh ( $3 \mu \mathrm{M}$ in puffer pipette); duration of puff is indicated by rectangular displacements of upper traces, which also show time marker $(1 \mathrm{~Hz})$. The pairs of records were made in the sequence shown: $a$ and $b$, in control perfusion solution; $c$ and $d$, in 3B (perfusion solution containing atropine sulfate, $1 \mu \mathrm{M}$, atenolol, $10 \mu \mathrm{M}$, and phentolamine, $0.1 \mu \mathrm{M}$ ); $e$ and $f$, after recovery in control solution; $g$ and $h$, in 8-phenyltheophylline [8-PT, less than $10 \mu \mathrm{M}$; see Materials and Methods, procedure (1)]; $i$ and $j$, after recovery in control solution. Voltage calibration applies to myocyte recordings.

of 8-PT $(g, h)$ was reciprocal to that of $3 \mathrm{~B}$; the neuron-evoked hyperpolarization was eliminated, but the effect of $\mathrm{ACh}$ was unchanged. The effects of 8-PT were readily reversed by perfusion with control medium $(i)$. The insensitivity to atropine of the neuron-evoked hyperpolarization and its abolition by 8-PT are consistent with its mediation by adenosine. The small myocyte depolarization evoked by neuronal stimulation in the presence of 8-PT ( $g$ ) was observed on several trials, and in one trial a longer train of neuronal impulses initiated beating by the myocytes. It seems likely that this was an adrenergic/purinergic dual-function neuron with a relatively weak adrenergic component.

Atropine-resistant hyperpolarizations were observed in 104 other microcultures, in various intensities; in 35 of these cases the neuron was adult derived. The effect of a methylxanthine was tested in almost every case. Usually, this was 8-PT, but theophylline was tested in four trials and 7-CET in three. With a small number of notable exceptions, the atropine-resistant hyperpolarizations were abolished or reduced. Because these methylxanthines are effective adenosine-receptor blockers in this concentration range (i.e., blockers of the $P_{1}$ purine receptors of Burnstock, 1978), but ineffective against ATP receptors (i.e., the $P_{2}$ receptors of Burnstock), the implication is that adenosine or AMP was responsible for the hyperpolarization; however, the secreted agent(s) might have been adenosine, a phosphorylated derivative, or a mixture of purines. In the cases in which a methylxanthine was not completely effective, it is possible that a higher concentration would have achieved complete block. However, there were five cases in which the usual concentration of 8-PT had little effect, and the existence of a third inhibitory transmitter must be considered; ATP, ADP, or both, acting via $\mathrm{P}_{2}$ receptors, are potentially of interest. 


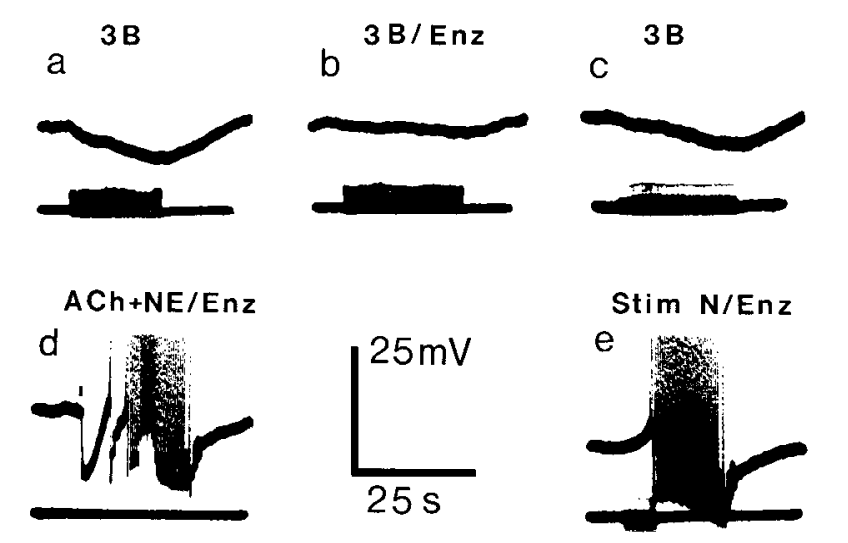

Figure 4. Effect of adenosine deaminase on an atropine-resistant hyperpolarization. Single-neuron microculture, neuron $49 \mathrm{~d}$ in vitro. In this and all subsequent figures, myocyte responses on upper traces; neuronal action potentials monitored at low gain on lower traces. Perfusion was with 3B (standard perfusion solution containing atropine sulfate, 1 $\mu \mathrm{M}$; atenolol, $10 \mu \mathrm{M}$; phentolamine, $0.1 \mu \mathrm{M}$ ) in $a$ and $c$; with 3B plus adenosine deaminase $(E n z ; 10 \mathrm{U} / \mathrm{ml})$ in $b$; and with control solution containing adenosine deaminase $(10 \mathrm{U} / \mathrm{ml})$ in $d$ and $e$. The perfusion medium contained $0.75 \%(\mathrm{vol} / \mathrm{vol})$ glycerol during each of the recordings $(a-e)$. Neuronal stimulation was at $16 \mathrm{~Hz}(a-c$ and $e)$. A brief puff of control solution containing ACh $(3 \mu \mathrm{M})$ and NE $(10 \mu \mathrm{M})$ was applied (at bar above upper trace) in $d$. Voltage calibration applies to upper (myocyte) traces; myocyte impulses in $d$ and $e$ are truncated.

In each of four experiments with theophylline (or with the theophylline/ethylenediamine complex, aminophylline) at 10 $\mu \mathrm{M}$, the atropine-insensitive response of the myocytes was reduced but not abolished. In two experiments, relatively weak and slowly developing myocyte hyperpolarizations (similar to that shown in Fig. $4 a$ ) were reduced by about $50 \%$. In another experiment, a large, rapidly developing, atropine-insensitive hyperpolarization was reduced by about $30 \%$ (Fig. $6, f$ and $g$ ). In most cases, 8-PT abolished or sharply reduced these responses and was clearly a more effective antagonist than theophylline in this system, as in others (e.g., Bruns, 1981). Furthermore, unlike theophylline, 8-PT has been reported to have little activity as a phosphodiesterase inhibitor (Smellie et al., 1979). Thus, both in terms of its apparent selectivity of action and its high affinity for adenosine receptors, 8-PT appears to be a useful diagnostic agent in this system, as in others where it has been examined.

In two experiments with 7-CET, relatively small myocyte hyperpolarizations were abolished by this compound at $10 \mu \mathrm{M}$; in a third experriment, a large hyperpolarization was reduced to about $25 \%$. On the basis of these limited tests, it is our impression that 7-CET is intermediate in its effectiveness between 8-PT and theophylline, as was found in another system (Bruns, 1981).

Two further lines of evidence are consistent with the idea that adenosine was responsible for the 8-PT-sensitive hyperpolarization: (1) The hyperpolarization could be partially or completely blocked by a solution containing adenosine deaminase (adenosine aminohydrolase, EC3.5.4.4.). This enzyme catalyzes the hydrolytic deamination of adenosine to inosine, which has been found to have no detectable effect on adenosine receptors (e.g., Axelsson and Holmberg, 1969; Burnstock et al., 1970; Hartzell, 1979), or in one system to have a slight excitatory effect where adenosine is inhibitory (Furukawa and Chiba, 1980). Its use in the present context was suggested to us by Dr. P. H. Patterson (see also, Akasu et al., 1984; Collis and Pettinger, 1982; Moody et al., 1984). The effect of this enzyme on the atropine-resistant inhibition was tested by adding it to the perfusion fluid, usually at $10-16 \mathrm{U} / \mathrm{ml}$. The enzyme preparation
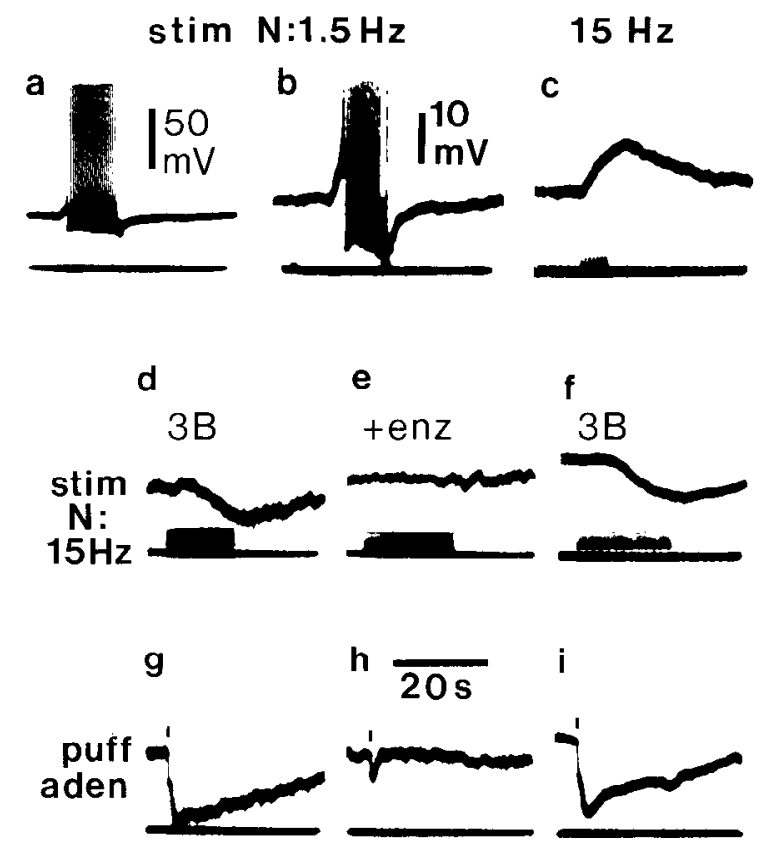

Figure 5. Comparison of the effect of adenosine deaminase on myocyte responses evoked by neuronal stimulation and by puffs of adenosine; solitary neuron $32 \mathrm{~d}$ in microculture. Perfusion was with standard perfusion solution in $a-c$; with perfusion solution plus $3 \mathrm{~B}$ (atropine sulfate, $1 \mu \mathrm{M}$; atenolol, $10 \mu \mathrm{M}$; phentolamine, $0.1 \mu \mathrm{M})$ in $d . f, g$, and $i$, and with perfusion solution plus $3 \mathrm{~B}$ and adenosine deaminase $(16 \mathrm{U} / \mathrm{ml})$ in $e$ and $h$. In $a$ and $b$, neuronal stimulation was at $1.5 \mathrm{~Hz}$ (10 stimuli); in $c-f$, at $15 \mathrm{~Hz}$ (for durations indicated). In $g-i$, puffs of adenosine $(10 \mu \mathrm{M}$ in pipette) are indicated by vertical bars above upper traces. Voltage calibration in $b$ applies to myocyte traces in $b-i$; myocyte impulses in $b$ are truncated. Time calibration in $h$ applies to all records.

that we have used (Sigma, type III) was reported by the supplier to be more than $5 \times 10^{5}$ times as active against adenosine as against AMP. (2) The 8-PT-sensitive hyperpolarization can be mimicked by puffs of adenosine $(2-10 \mu \mathrm{M})$, and this effect is sensitive to methylxanthines or adenosine deaminase.

\section{The effect of adenosine deaminase}

As illustrated in Figure 4, in an experiment on a singlc-ncuron microculture, a slowly developing, atropine-resistant hyperpolarization was evoked by prolonged stimulation of the neuron in the presence of $3 \mathrm{~B}(a)$. (As in many other microcultures, the hyperpolarization developed along a biphasic time course; we have not investigated the origin of this effect.) Perfusion with adenosine deaminase (Sigma, type III; $10 \mathrm{U} / \mathrm{ml}$ ), still in the presence of $3 \mathrm{~B}$, nearly abolished the response $(b)$. Perfusion with enzyme-free 3B solution brought about partial restoration of the response; in other experiments the reversal of the enzyme effect was complete. Glycerol was added to the enzyme-free solutions in the experiment of Figure 4, so that all solutions contained glycerol at $0.75 \%$ (vol/vol; see Materials and Methods). Earlier in this experiment it was found that aminophylline $(10 \mu \mathrm{M})$ delayed the onset of the atropine-resistant hyperpolarization and reduced its final amplitude by about $50 \%$, providing additional evidence that this response was mediated by adenosine. Note that the very much higher activity of the enzyme against adenosine than against AMP favors the idea that the active agent was adenosine rather than AMP, another agonist of $P_{1}$-receptors blocked by methylxanthines.

The action of the adenosine deaminase solution was specific to the extent that it did not seem to impair either the response of the myocytes to the other transmitters or the release of the other transmitters by the neuron. In the presence of the enzyme 
(but in the absence of 3B), the myocytes gave their characteristic responses to brief puffs of $\mathrm{ACh}$ and NE (Fig. 4d). Furthermore, this neuron also had a component of adrenergic function that continued to be effective in the presence of the enzyme (in the absence of 3B; Fig. $4 e$ ).

Tests with adenosine deaminase $(10-16 \mathrm{U} / \mathrm{ml})$ were made on 16 single-neuron microcultures; the neurons were neonate derived in 9 of these cases, adult-derived in the other 7 . In each case, the atropine-resistant hyperpolarization was reduced (by $30-100 \%$ ). In 8 of these cases, control trials were made for the effect of the glycerol in the enzyme solution, and no effect attributable to glycerol was observed.

Evidence that adenosine deaminase blocked responses to puffs of adenosine is shown in Figure 5. Stimulation of the solitary neuron ( $32 \mathrm{~d}$ in microculture) at $1.5 \mathrm{~Hz}$ evoked a period of beating in the myocytes $(a, b)$. With stimulation at $15 \mathrm{~Hz}$, the myocytes were depolarized but did not beat; their response was apparently damped $(c)$. Perfusion with $3 \mathrm{~B}$ solution converted the response to a hyperpolarization, unmasked when the adrenergic depolarization was blocked $(d)$. Addition of adenosine deaminase to the 3B solution (e) eliminated the hyperpolarization, which reappeared when the enzyme was washed out with $3 \mathrm{~B}$ solution $(f)$. In parallel observations, a brief puff of adenosine $(10 \mu \mathrm{M})$ hyperpolarized the myocytes by $12-15 \mathrm{mV}$ for about $30 \mathrm{sec}(g, i)$; in the presence of adenosine deaminase, the puffed adenosine was ineffective $(h)$. These observations illustrate the sensitivity of the myocytes to adenosine and indicate that the enzyme preparation worked effectively against authentic adenosine, as well as against the agent mediating the neuron-induced effect.

In the presence of 8-PT, as in the presence of adenosine deaminase, the effect of puffed adenosine was always reduced or eliminated. However, in a few preliminary trials, the effect of puffed ATP was sometimes reduced by 8-PT and sometimes hardly changed. ATP-evoked hyperpolarizations that were resistant to 8-PT raise the possibility of a second purinergic receptor (e.g., the $\mathrm{P}_{2}$-receptor; Burnstock, 1976). Nevertheless, in most microcultures the atropine-resistant response was abolished or sharply reduced by 8-PT or adenosine deaminase (or a combination of the two). While it is likely that the active agent was primarily adenosine, the effect is hereafter called purinergic (rather than adenosinergic) to acknowledge the possibility that ATP (or ADP) is the major secreted agent.

One of the most intense and rapid purinergic effects observed in a single-neuron microculture is illustrated in Figure 6 . In the presence of $3 \mathrm{~B}$, a single neuronal impulse (at the arrow in $a$ ) evoked an appreciable hyperpolarization (ca. $7 \mathrm{mV}$ ). Two impulses gave rise to a slightly larger response; a low-frequency train of impulses (at $1.5 \mathrm{~Hz}$ ) maintained a substantial membrane hyperpolarization throughout the period of stimulation $(b)$. The response latency, measured from the peak of the single neuronal action potential to the first detectable departure of the myocyte membrane potential from the baseline $(c)$, was about $150 \mathrm{msec}$. This was the shortest latency observed for the atropine-resistant hyperpolarization; as with the cholinergic and adrenergic responses, the apparent latency was longer and the time course slower when the response was weaker. In several other experiments, small myocyte hyperpolarizations (ca. 1-4 mV) were evoked by single neuronal impulses. Most purinergic neurons evoked a response in the myocytes only when activated with a train of stimuli (e.g., Figs. 4, 5), and in some of these cases, a relatively intense adrenergic or cholinergic effect masked a slowly rising purinergic effect, as in Figure 5.

The atropine-resistant hyperpolarizations of Figure $6, a-c$, were identified as purinergic by their sensitivity to adenosine deaminase and theophylline. These tests, illustrated in $d-k$, were begun about $40 \mathrm{~min}$ after the recordings in $a$; during this interval, the control responses (in 3B) to single impulses had be-
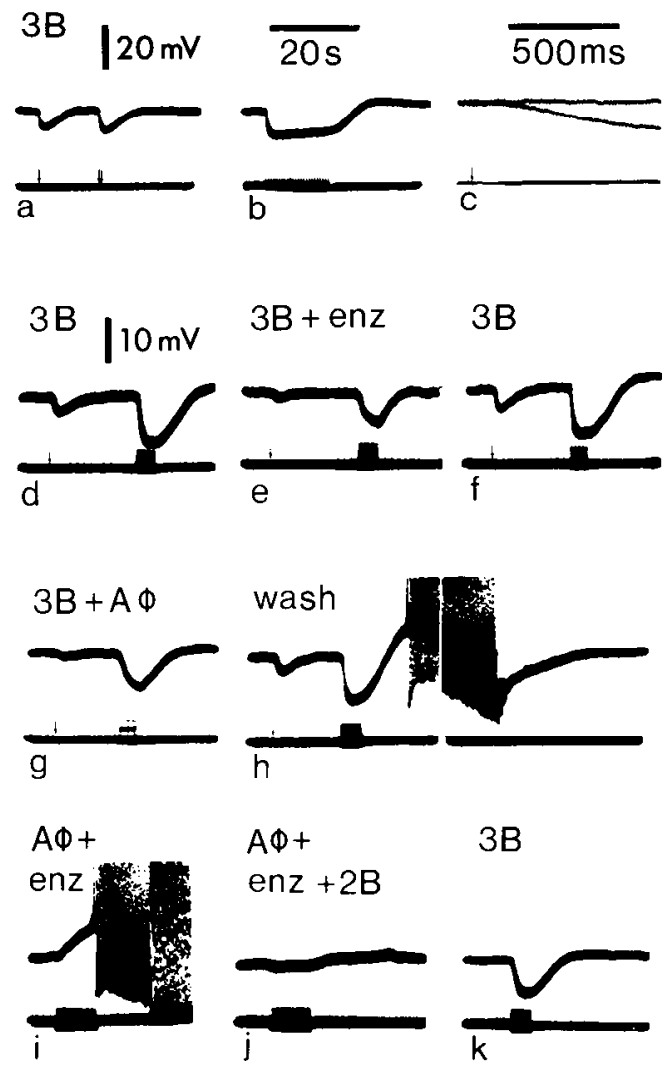

Figure 6. Strong, rapidly rising purinergic response. Solitary neuron $49 \mathrm{~d}$ in vitro (same culture dish as microculture of Fig. 4). The initial recordings from this neuron $(a-c)$ were made in $3 \mathrm{~B}(1 \mu \mathrm{M}$ atropine sulfate, $10 \mu \mathrm{M}$ atenolol, $0.1 \mu \mathrm{M}$ phentolamine); see response later recorded in standard perfusion solution in $h$. 3B was also present in $d-g$ and $k$; in addition, the solution contained adenosine deaminase $(10$ $\mathrm{U} / \mathrm{ml}$ ) in $e$ and aminophylline ( $A \phi$; equivalent to $10 \mu \mathrm{M}$ theophylline) in $g$. The solution contained aminophylline and adenosine deaminase (without $3 \mathrm{~B}$ ) in $i$, and aminophylline, adenosine deaminase, atenolol $(10 \mu \mathrm{M})$, and phentolamine $(0.1 \mu \mathrm{M})$ in $j$. Single neuronal stimuli were applied at the arrows (lower traces) in $a, c$, and $d-h$; trains of stimuli were at $1.5 \mathrm{~Hz}$ in $b$ and at $8 \mathrm{~Hz}$ in $d-k$. Voltage calibrations for myocyte traces only: that in $a$ applies to $a$ and $b$; that in $d$ applies to $c-k$; time calibration in $b$ applies to all records but $c$.

come smaller (note change in amplification). Each sweep in $d-$ $h$ shows the myocyte responses to a single neuronal impulse and to a train of impulses. Adenosine deaminase $(10 \mathrm{U} / \mathrm{ml})$, in the presence of $3 \mathrm{~B}$, reduced the myocyte response evoked by a single neuronal impulse to about one-third of control amplitude; it reduced the larger response evoked by the train of impulses to about two-thirds of control amplitude and slowed its rising phase $(e)$. These effects were fully reversible $(f)$. The relatively weaker effect of the enzyme in this experiment compared to that of Figure 4 may have been caused by the presence of a higher concentration of adenosine or the additional presence of a phosphorylated derivative resistant to the enzyme action.

The effect on the hyperpolarization of aminophylline (equivalent to $10 \mu \mathrm{M}$ theophylline) was also tested in this experiment. Aminophylline in $3 \mathrm{~B}$ reduced the myocyte responses (Fig. $6 \mathrm{~g}$ ) by roughly the same amount as had adenosine deaminase. The preparation was then washed with drug-free perfusion medium. The amplitudes of the hyperpolarizations in control medium $(h)$ and in $3 \mathrm{~B}(f)$ were about the same; however, in the absence of $3 \mathrm{~B}$ an excitatory, adrenergic component was revealed (depolarization and a burst of beating following the hyperpolarization in $h$ ). When adenosine deaminase and theophylline were applied to this microculture simultaneously (without $3 \mathrm{~B}$ ), they 

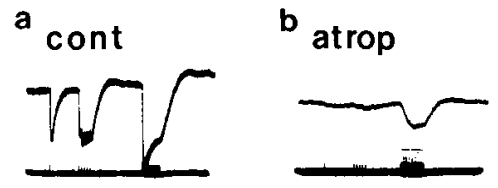

C atrop
$+8 \mathrm{PT}$
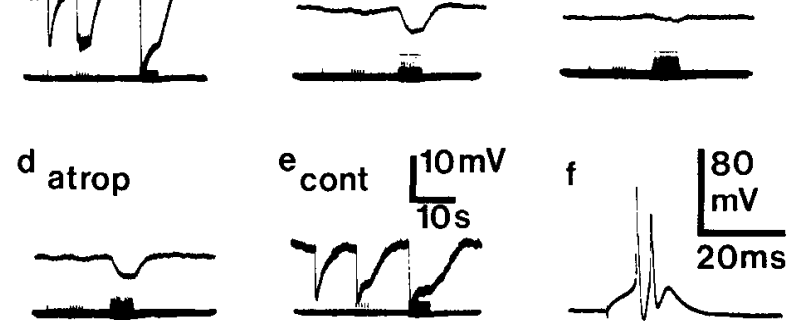

Figure 7. Cholinergic and purinergic responses evoked by a solitary neuron ( $42 \mathrm{~d}$ in microculture). Perfusion in $a, e$, and $f$ was with standard solution; in $b$ and $d$ with standard solution containing atropine sulfate $(1 \mu \mathrm{M})$; and in $c$ with standard solution containing atropine sulfate and 8-PT (nominally $1 \mu \mathrm{M})$. In addition, the perfusion solution in $a-e$ contained hexamethonium $(1 \mu \mathrm{M})$. In each record $(a-e)$, the neuron was activated with a single stimulus, then with 5 stimuli at $1.5 \mathrm{~Hz}$, and finally with 80-100 stimuli at $16 \mathrm{~Hz}$. The recording from the neuron at higher sweep speed $(f)$ illustrates the autaptic EPSPs, which provided additional evidence for cholinergic function; the sensitivity of the EPSPs in this neuron to hexamethonium is illustrated in Figure 5 of Furshpan et al. (1986). The calibrations in $e$ apply to $a-e$ (voltage calibration applies to upper traces only).

were much more effective than either agent applied alone. Together they converted the initial response from a hyperpolarization $(h)$ to a depolarization $(i)$. Even when the adrenergic depolarization was nearly eliminated by atenolol and phentolamine (2B), only a small hyperpolarization remained in the presence of the enzyme and aminophylline $(j)$. This indicates that there was no substantial cholinergic component in the transmitter repertoire of this neuron and that the hyperpolarization was largely or entirely purinergic. It also suggests that the purinergic effect was mediated by $P_{1}$ receptors (e.g., by adenosine or adenosine and AMP).

In each of the cases illustrated above (Figs. 1 and 3-6), the neuron appeared to be adrenergic/purinergic. However, we have observed neurons (more than 75) in which cholinergic and purinergic functions were combined (often in addition to adrenergic function and/or the nonadrenergic excitation described in the next paper of this series: Matsumoto et al., in press). The responses of a cholinergic/purinergic neuron (in a singlc-ncuron microculture) are shown in Figure 7. Each of the recordings in $a-e$ shows the responses of the myocytes to a single neuronal impulse and to trains of impulses at 1.5 and $16 \mathrm{~Hz}$. In control solution, the large, rapid response to a single impulse was characteristic of strong cholinergic function (Furshpan et al., 1986). In the presence of atropine, only the $16 \mathrm{~Hz}$ stimulus evoked an appreciable response. This residual response was nearly abolished by addition of 8-PT, indicating that it was substantially or entirely purinergic. The drug effects were largely reversible $(d, e)$. Autaptic EPSPs in the neuron $(f)$ confirmed the presence of cholinergic function (Furshpan et al., 1986). In this case, the cholinergic response appeared to be much stronger than the purinergic response. In other experiments the reverse was true. Similar variability in the relative strengths of adrenergic and purinergic effects were observed. For example, in Figure 3 the adrenergic effects were relatively weak; in Figures 4 and 5, the purinergic effects were relatively weak; in Figure 6 both effects were strong.

In three experiments, one on an adult-derived neuron, the other two on neonate-derived neurons, the only observable effect of neuronal stimulation was purinergic. The assay of the adult-derived neuron is shown in Figure 8 . The myocyte hyperpolarization evoked in control medium $(a)$ was abolished by 8-PT $(b)$. To test the possibility of a null response due to si-

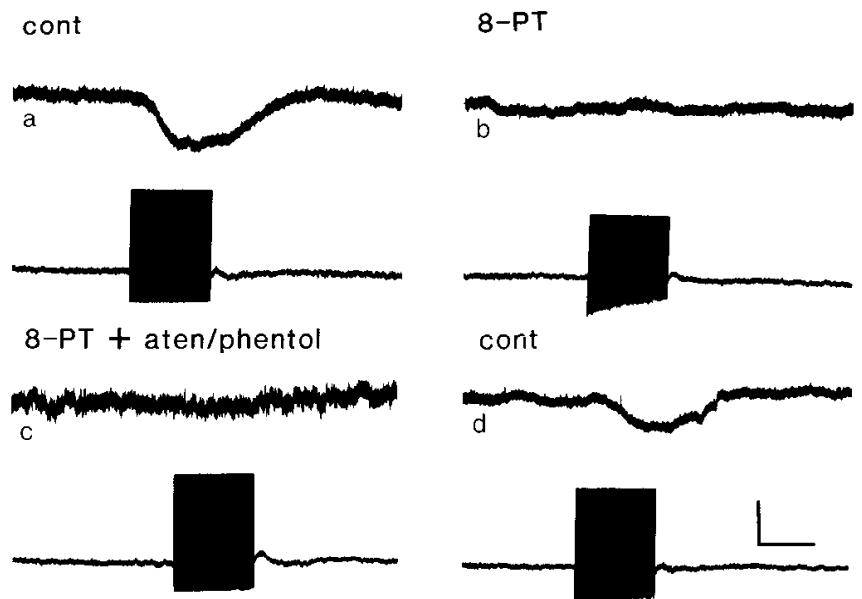

Figure 8. A solitary adult-derived neuron, apparently only purinergic, $57 \mathrm{~d}$ in microculture. Perfusion in $a$ and $d$ was with standard solution in $b$, with standard solution containing 8-PT $[<10 \mu \mathrm{M}$; see Materials and Methods, procedure (1)]; in $c$, with standard solution containing 8-PT plus atenolol $(10 \mu \mathrm{M})$ and phentolamine $(0.1 \mu \mathrm{M})$. Stimuli were applied to the neuron at $20 \mathrm{~Hz}$. Voltage calibration: $4 \mathrm{mV}$ for $a, b$, and $d ; 2 \mathrm{mV}$ for $c$. Time calibration, $10 \mathrm{sec}$.

multaneous adrenergic and purinergic effects, atenolol and phentolamine were added to the 8-PT, but this failed to unmask any 8-PT-resistant response ( $c$; note increased gain). Recovery of the response, after washout of the blockers, was incomplete (d).

The incidence of purinergic function and the pattern of its occurrence in combination with other responses evoked by solitary neurons are discussed in a forthcoming paper (Matsumoto et al., in press). It may be mentioned here that we observed purinergic function in about half of these neurons. In one sample of 303 microcultures, $46 \%$ of the neonate-derived neurons (102 of 223 ) and $48 \%$ of the adult-derived neurons (38 of 80 ) exhibited purinergic function.

\section{Discussion}

Several lines of evidence are consistent with the idea that the atropine-resistant hyperpolarizations described here were mediated, at least in large part, by adenosine: (1) The neuronally evoked effect was strongly or completely blocked by methylxanthines at concentrations reported to block adenosine receptors in other preparations (e.g. Bruns, 1981; Smellie et al., 1979). (2) A specific degradative enzyme, adenosine deaminase, was also an effective antagonist. (3) These agents exhibited specificity of action to the extent that they failed to block adrenergic and cholinergic responses. (4) Puffed adenosine mimicked the neuronally evoked response in the $1-10 \mu \mathrm{M}$ concentration range; the response to puffed adenosine was strongly or completely blocked by the methylxanthines or adenosine deaminase at the same concentrations that affected the neuronally evoked response.

Responses that were nearly or completely abolished by adenosine deaminase (e.g., Figs. 4 and 5) were presumably evoked predominantly or entirely by adenosine. Responses that required an adenosine-receptor antagonist in addition to the enzyme to achieve an effective block (e.g., Fig. 6) may have been evoked by adenosine at higher concentrations than could be quickly degraded by the enzyme or by the additional presence of adenine nucleotides against which the enzyme has very low activity. In either case, the responses were presumably mediated by $P_{1}$ receptors (Burnstock, 1978; see Collis and Pettinger, 1982; Moody et al., 1984).

There is evidence for vesicular storage of ATP in some sympathetic neurons, for secretion of ATP in some sympathetically innervated tissues (see introduction), and for the storage of ATP 
in sympathetic neurons in mass culture (Wolinsky and Patterson, 1985). There is also extensive evidence for the presence of extracellular nucleotidases in tissues (e.g., Mannery and Dryden, 1979) and for the degradation of secreted ATP to AMP and to adenosine and its metabolites (see introduction). It is plausible that the neurons in microculture secreted ATP that was degraded to AMP and/or adenosine, which then interacted with the myocyte receptors. However, it is also possible that a special, presumably small (Wolinsky and Patterson, 1985), pool of adenosine, or AMP, was selectively released by the neuronal impulses.

It is natural to suppose that the active purine or its precursor, released by neuronal stimulation, was secreted by the neuron. However, the microculture experiments do not formally distinguish secretion by the neuron from secretion by the cardiac cells triggered by some other (nonelectrogenic) neuronal agent. In fact, there is much evidence for release of adenosine from a variety of active tissues, including heart cells (for reviews, see Berne et al., 1971, 1983). The vasodilatory action of adenosine in many tissues led Berne and colleagues to propose that the released adenosine mediates a homeostatic control of local circulation. According to this hypothesis, when working cells break down ATP faster than it can be oxidatively resynthesized, adenosine is released to increase local blood flow, thus linking metabolic support to metabolic stress. It is plausible that cells in microculture also release adenosine in response to metabolic stress; however, it seems unlikely that such release accounts for most of the purinergic effects described in this paper. Purinergic inhibition was often the first detectable response of the myocyte (Figs. 1, 2d, 3a, 6h) and occasionally the only response (Fig. 8). Thus, the inhibition appeared to be a direct result of neuronal impulses rather than a consequence of prior myocyte activity. Furthermore, pronounced purinergic responses occasionally followed single neuronal impulses (Fig. $6 a$ ) that did not plausibly stress either the neuron or the myocytes metabolically. In some cases (Fig. 4a), purinergic hyperpolarizations rose gradually during prolonged neuronal stimulation that might have induced metabolic stress; yet, in other cases, similar episodes of stimulation failed to evoke purinergic inhibition (e.g., Fig. $7 b$ of Furshpan et al., 1986). It is unlikely that adenosine release was caused simply by hyperpolarization of the myocytes; similar hyperpolarizations evoked by ACh (opening similar or identical channels; Belardinelli and Isenberg, 1983) lacked a detectable purinergic component. Evidence for a $\mathrm{K}^{+}$-evoked release of purines by sympathetic neurons grown in mass cultures, in the virtual absence of nonneuronal cells, was obtained by Wolinsky and Patterson (1985). This clearly favors attribution of the purinergic effect in microcultures to secretion of purine(s) from the ncuron, pending demonstration that purine relcasc in mass culture is quantitatively sufficient to account for the observed effects in microcultures.

These considerations lead us to the following tentative conclusions: Many of the neurons in microculture released purine(s) as at least one of their synaptic transmitters. Perhaps some release of purine was nonsynaptic and was due to metabolic stress of the neuron. It is also possible that, as one of their synaptic functions, sympathetic neurons release purines in vivo to help target tissues cope with metabolic stress (e.g., dilation of blood vessels; see Muramatsu et al., 1981).

Release of purine(s) from synaptic vesicles would presumably be $\mathrm{Ca}^{2+}$-dependent. In a single experiment, a purinergic hyperpolarization, and an accompanying adrenergic excitation, were both abolished by a medium with reduced $\mathrm{Ca}^{2+}(0.15 \mathrm{~mm})$ and increased $\mathrm{Mg}^{2+}(10 \mu \mathrm{M})$. It appears not to be known whether release of adenosine during metabolic stress occurs via a $\mathrm{Ca}^{2+}$. dependent mechanism.

Is there an obligatory coupling in these neurons between the release of purine(s) and the release of other transmitters, as might occur if they were co-stored in the same vesicles at constant stoichiometric ratio? The presence in most microcultures of only a single neuron was helpful in addressing this question. In about one-half of such cases the neurons released NE and/or ACh in the absence of detectable purine release. Conversely, in a few cases the neurons were apparently purely purinergic (although we could not exclude the possibility that these neurons released another agent or agents to which the myocytes gave no electrical response). Even in those cases (ca. $50 \%$ ) in which purine was co-released, the adrenergic, cholinergic, and purinergic components of composite myocyte responses appeared to vary greatly in their relative strengths from neuron to neuron; in the extreme, each of these functions was apparently expressed in isolation (Furshpan et al., 1986, and this paper). From the type of evidence illustrated in Figures 2 and 3, it seems likely that these variations primarily reflected differences in neuronal transmitter status and not in the responsiveness of the myocytes (cf. evidence for variation of adrenergic and cholinergic states in Potter et al., 1986, e.g., Fig. 8). This suggests that NE and purine, or $\mathrm{ACh}$ and purine, were not co-released at constant stoichiometric ratio and, assuming exocytotic release, that they were not co-stored in the releasable vesicles at constant ratio. It does not exclude co-storage in the same vesicles but in varying proportions.

The fact that purinergic effects appeared to vary independently of adrenergic or cholinergic effects, and over as wide a range, raises the possibility of independent control mechanisms for the expression of purinergic function. However, we have little indication of what factors might exert such control; the proportion of neurons displaying purinergic function appears to be somewhat reduced in high- $\mathrm{K}^{+}$medium, which depresses the adrenergic-to-cholinergic transition induced by heart-cell factor(s) (Potter et al., 1986; Walicke et al., 1977), but the sample was relatively small and the effect was not dramatic (see Matsumoto et al., in press).

Burnstock and colleagues have suggested that the terminals of some purinergic neurons can be recognized in the EM by their content of "large opaque vesicles" (e.g., Baumgarten et al., 1970; see Burnstock, 1972). Dr. S. Landis (personal communication) has not detected such vesicles in the terminals of microcultured sympathetic neurons found to be purinergic in the myocyte assay.

If purinergic function is exhibited by sympathetic neurons in vivo, it would plausibly be target-specific. The frequent finding of purinergic inhibition of cardiac myocytes in culture, where neurons and heart cells have been arbitrarily mixed, implies nothing about purinergic function in the normal innervation of the heart. Hartzell (1979) observed large hyperpolarizations of the isolated sinus venosus of the frog in response to adenosine and adenine nucleotides. However, he could not detect any purinergic response to stimulation of nerves supplying the sinus (although other conditions of stimulation might reveal such an effect).

A variety of evidence indicates that catecholamine effects on the heart are mediated by cAMP (e.g., Tsien, 1977). In contrast, recent observations with patch-clamp techniques suggest that cholinergic responses of rabbit atrial cells do not depend on freely diffusible messenger molecules (Soejima and Noma, 1984). It is unclear whether the action of purines on heart-cell channels is direct or second-messenger mediated. The minimal latency we observed for the purinergic response (about $150 \mathrm{msec}$ ) was intermediate between the minimal latencies observed for the cholinergic (35 $\mathrm{msec}$ ) and adrenergic responses $(350 \mathrm{msec}$; Furshpan et al., 1986). A major effect of adenosine on atrial myocytes is the activation of a set of inwardly rectifying $\mathrm{K}^{+}-$ channels that closely resemble the channels activated by $\mathrm{ACh}$ (Belardinelli and Isenberg, 1983); however, it is not yet clear whether the same set of channels is activated by both agents. 
The experiments described here have provided clear evidence for a purinergic (adenosinergic) effect mediated by sympathetic neurons in vitro; it seems likely that the purinergic agent was released primarily from the neurons rather than from the myocytes, but uncertainty on this point remains. It seems very likely from the evidence reviewed in the introduction (e.g., Fedan et al., 1982; Muramatsu et al., 1981; Sneddon and Burnstock, 1984, 1985; Westfall et al., 1983) that some sympathetic neurons evoke purinergic responses in vivo (although the greater complexity of the intact tissues increases the uncertainty about the source of the purines). These responses appeared to be mediated primarily by ATP (i.e., by $P_{2}$ receptors). The present experiments indicate that sympathetic neurons can mediate adenosinergic responses as well.

\section{References}

Akasu, T., P. Shinnick-Gallagher, and J. P. Gallagher (1984) Adenosine mediates a slow hyperpolarizing synaptic potential in autonomic neurones. Nature 311: 62-65.

Axelsson, J., and R. Holmberg (1969) The effects of extracellularly applied ATP and related compounds on the electrical and mechanical activity of the smooth muscle of taenia coli of the guinea pig. Acta Physiol. Scand. 75: 149-156.

Baer, H. P., and G. I. Drummond (1979) Physiological and Regulatory Functions of Adenosine and Adenosine Nucleotides, Raven, New York.

Banks, P. (1966) The release of adenosine triphosphate catabolites during the secretion of catecholamines by bovine adrenal medulla. Biochem. J. 101: 536-541.

Baumgarten, H. G., A. F. Holstein, and C. Owman (1970) Auerbach's plexus of mammals and man: Electron microscopic identification of three different types of neuronal processes in myenteric ganglia of the large intestine from Rhesus monkeys, guinea pigs and man. Z. Zellforsch. Mikroscop. Anat. 106: 376-397.

Belardinelli, L., and G. Isenberg (1983) Isolated atrial myocytes: Adenosine and acetylcholine increase potassium conductance. Am. J. Physiol. 244: H734-H737.

Berne, R. M. (1963) Cardiac nucleotides in hypoxia: Possible role in regulation of coronary blood flow. Am. J. Physiol. 204: 317-322.

Berne, R. M., R. Rubio, J. G. Dobson, and R. R. Curnish (1971) Adenosine and adenine nucleotides as possible mediators of cardiac and skeletal muscle blood flow regulation. Circ. Res. (Suppl. 1) 28/ 29: 115-119.

Berne, R. M., T. W. Rall, and R. Rubio (1983) Regulatory Function of Adenosine, Martinus Nijhoff, Boston.

Blaschko, H., G. V. R. Born, A. D'Iorio, and N. R. Eade (1956) Observations on the distribution of catecholamines and adenosinetriphosphate in the bovine adrenal medulla. J. Physiol. (Lond.) 133: 548-557.

Bruns, R. F. (1981) Adenosine antagonism by purines, pteridines and benzopteridines in human fibroblasts. Biochem. Pharmacol. 30:325333.

Burnstock, G. (1971) Neural nomenclature. Nature 229: 282.

Burnstock, G. (1972) Purinergic nerves. Pharmacol. Rev. 24: 509_ 581.

Burnstock, G. (1976) Purinergic receptors. J. Theor. Biol. 62: 491503.

Burnstock, G. (1978) A basis for distinguishing two types of purinergic receptor. In Cell Membrane Receptors for Drugs and Hormones, R. W. Straub and L. Bolis, eds., pp. 107-1 18, Raven, New York.

Burnstock, G. (1979) Past and current evidence for the purinergic nerve hypothesis. In Physiological and Regulatory Functions of Adenosine and Adenine Nucleotides, H. P. Baer and G. I. Drummond, eds., pp. 3-32, Raven, New York.

Burnstock, G. (1980) Purinergic receptors in the heart. Circ. Res. (Suppl. I) 46: I-175-I-182.

Burnstock, G., and P. Meghji (1981) Distribution of $P_{1}-$ and $P_{2}-p u-$ rinoceptors in the guinea-pig and frog heart. Br. J. Pharmacol. 73: 879-885.

Burnstock, G., and P. Meghji (1983) The effect of adenyl compounds on the rat heart. Br. J. Pharmacol. 79: 211-218.

Burnstock, G., G. Campbell, D. Satchell, and A. Smythe (1970) Evidence that adenosine triphosphate or a related nucleotide is the transmitter substance released by non-adrenergic inhibitory nerves in the gut. Br. J. Pharmacol. 40: 668-688.

Choi, D., and G. D. Fischbach (1981) GABA conductance of chick spinal cord and dorsal root ganglion neurons in cell culture. J. Neurophysiol. 45: 605-620.

Collis, M. G., and S. J. Pettinger (1982) Can ATP stimulate $P_{1}$-receptors in guinea-pig atrium without conversion to adenosine? Eur. J. Pharmacol. 81: 521-529.

Douglas, W. W., and A. M. Poisner (1966a) Evidence that the secreting adrenal chromaffin cell releases catecholamines directly from ATPrich granules. J. Physiol. (Lond.) 183: 236-248.

Douglas, W. W., and A. M. Poisner (1966b) On the relation between ATP splitting and secretion in the adrenal chromaffin cell: Extrusion of ATP (unhydrolysed) during release of catecholamines. J. Physiol. (Lond.) 183: 249-256.

Drury, A. N., and A. Szent-Gyorgyi (1929) The physiological activity of adenine compounds with essential reference to their action upon the mammalian heart. J. Physiol. (Lond.) 68: 213-237.

Fedan, J. S., G. K. Hogaboom, J. P. O'Donncll, J. Colby, and D. P. Westfall (1981) Contribution by purines to the neurogenic response of the vas deferens of the guinea pig. Eur. J. Pharmacol. 69: 41-53.

Fedan, J. S., G. K. Hogaboom, D. P. Westfall, and J. P. O'Donnell (1982) Comparison of the effects of arylazido aminopropionyl ATP $\left(\mathrm{ANAPP}_{3}\right.$ ), an ATP antagonist, on responses of the smooth muscle of the guinea-pig vas deferens to ATP and related nucleotides. Eur. J. Pharmacol. 85: 277-290.

Fried, G. (1980) Small noradrenergic storage vesicles isolated from rat vas deferens-biochemical and morphological characterization. Acta Physiol. Scand. (Suppl.) 493: 1-28.

Furshpan, E. J., P. R. MacLeish, P. H. O'Lague, and D. D. Potter (1976) Chemical transmission between rat sympathetic neurons and cardiac myocytes developing in microculture: Evidence for cholinergic, adrenergic, and dual-function ncurons. Proc. Natl. Acad. Sci. USA 73 : 4225-4229.

Furshpan, E. J., D. D. Potter, and S. C. Landis (1982) On the transmitter repertoire of sympathetic neurons in culture. Harvey Lectures Ser. 76: 149-191.

Furshpan, E. J., S. C. Landis, S. G. Matsumoto, and D. D. Potter (1986) Synaptic functions in rat sympathetic neurons in microcultures. I. Secretion of norepinephrine and acetylcholine. J. Neurosci. 6: 10611079.

Furukawa, Y, and S. Chiba (1980) Inotropic and chronotropic responses to inosine in isolated and blood perfused dog atria. Eur. J. Pharmacol. 67: 339-345.

Hartzell, H. C. (1979) Adenosine receptors in frog sinus venosus: Slow inhibitory potentials produced by adenine compounds and acetylcholine. J. Physiol. (Lond.) 293: 23-49.

Hillarp, N- $\AA$., and G. Thieme (1959) Nucleotides in the catecholamine granules of the adrenal medulla. Acta Physiol. Scand. 45: 155-167.

Holton, F. A., and P. Holton (1954) The capillary dilator substances in dry powders of spinal roots: A possible role of ATP in chemical transmission from nerve endings. J. Physiol. (Lond.) 126: 124-140.

Holton, P. (1959) The liberation of adenosine triphosphate on antidromic stimulation of sensory nerves. J. Physiol. (Lond.) 145: 494504.

Mannery, J. F., and E. E. Dryden (1979) Ecto-enzymes concerned with nucleotide metabolism. In Physiological and Regulatory Functions of Adenosine and Adenine Nucleotides, H. P. Baer and G. I. Drummond, eds., pp. 323-339, Raven, New York.

Matsumoto, S. G., D. Sah, D. D. Potter, and E. J. Furshpan (in press) Synaptic functions in rat sympathetic principal neurons grown in microcultures. IV. A slow excitatory effect on cardiac myocytes and the variety of multiple transmitter states. J. Neurosci.

Moody, C. J., P. Meghji, and G. Burnstock (1984) Stimulation of $P_{1^{-}}$ purinoceptors by ATP depends partly on its conversion to AMP and adenosine and partly on direct action. Eur. J. Pharmacol. 97: 47-54. Muramatsu, I., M. Fujiwara, A. Miura, and Y. Sakakibara (1981) Possible involvement of adenine nucleotides in sympathetic neuroeffector mechanisms of dog basilar artery. J. Pharmacol. Exp. Ther. 216: 401409.

Paddle, B. M., and G. Burnstock (1974) Release of ATP from perfused heart during coronary vasodilation. Blood Vessels $11: 110-119$

Phillis, J. W., and P. H. Wu (1981) The role of adenosine and its nucleotides in central synaptic transmission. Prog. Neurobiol. 16: 187-239.

Phillis, J. W., J. P. Edstrom, G. K. Kostopoulos, and J. R. Kirkpatrick (1979) Effects of adenosine and adenine nucleotides on synaptic transmission in the cerebral cortex. Can. J. Physiol. Pharmacol. 57. 1289-1312.

Potter, D. D., E. J. Furshpan, and S. C. Landis (1983) Transmitter 
status in cultured rat sympathetic neurons: Plasticity and multiple function. Fed. Proc. 42: 1626-1632.

Potter, D. D., S. C. Landis, S. G. Matsumoto, and E. J. Furshpan (1986) Synaptic functions in rat sympathetic neurons in microcultures. II. Adrenergic/cholinergic dual status and plasticity. J. Neurosci. 6: 10801098.

Ribeiro, J. A. (1979) Purinergic modulation of transmitter release. J. Theor. Biol. 80: 259-270.

Schrader, J., E. Gerlach, and G. Baumann (1979) Sites and mode of action of adenosine in the heart. II. Ventricular myocardium. In Physiological and Regulatory Functions of Adenosine and Adenine $\mathrm{Nu}$ cleotides, H. P. Baer and G. I. Drummond, eds., pp. 137-144, Raven, New York.

Silinsky, E. M. (1975) On the association between transmitter secretion and the release of adenine nucleotides from mammalian motor nerve terminals. J. Physiol. (Lond.) 247: 145-162.

Smellie, F. W., C. W. Davis, J. W. Daly, and J. N. Wells (1979) Alkylxanthines: Inhibition of adenosine-elicited accumulation of cyclic AMP in brain slices and of brain phosphodiesterase activity. Life Sci. 24: $2475-2482$.

Sneddon, P., and G. Burnstock (1984) Inhibition of excitatory junction potentials in guinea-pig vas deferens by $\alpha, \beta$-methylene ATP: Further evidence for ATP and noradrenaline as cotransmitters. Eur. J. Pharmacol. 100: 85-90.

Sneddon, P., and G. Burnstock (1985) ATP as a co-transmitter in rat tail artery. Eur. J. Pharmacol. 106: 149-152.

Sneddon, P., D. P. Westfall, and J. S. Fedan (1982) Cotransmitters in the motor nerves of the guinea-pig vas deferens: Electrophysiological evidence. Science 218: 693-695.

Soejima, M., and A. Noma (1984) Mode of regulation of the AChsensitive K-channel by the muscarinic receptor in rabbit atrial cells. Pfluegers Arch. 400: 424-431.
Stevens, P., R. L. Robinson, K. Van Dyke, and R. Stitzel (1975) Synthesis, storage and drug-induced release of ATP-8 $-{ }^{3} \mathrm{H}$ in the perfused bovine adrenal gland. Pharmacology (Basel) 13: 40-55.

Stone, T. W. (1981) Physiological roles for adenosine and ATP in the nervous system. Neuroscience 6: 523-555.

Theobald, R. J. (1983) The effect of arylazidoaminoproprionyl ATP on atropine resistant contractions of the cat urinary bladder. Life Sci. 32: $2479-2484$.

Tsien, R. W. (1977) Cyclic AMP and contractile activity in heart. Adv. Cyclic Nucleotide Res. 8: 363-420.

Van Dyke, K., R. Robinson, P. Urquilla, D. Smith, M. Taylor, M. Trush, and M. Wilson (1977) An analysis of nucleotides and catecholamines in bovine medullary granules by anion exchange high pressure liquid chromatography and fluorescence. Pharmacology (Basel) $15: 377-391$

Wagner, J. A., S. S. Carlson, and R. B. Kelly (1978) Chemical and physical characterization of cholinergic synaptic vesicles. Biochemistry 17: 1199-1206.

Walicke, P. A., R. B. Campenot, and P. H. Patterson (1977) Determination of transmitter function by neuronal activity. Proc. Natl. Acad. Sci. USA 74: 5767-5771.

Westfall, D. P., J. S. Fedan, J. Colby, G. K. Hogaboom, and J. P. O'Donnell (1983) Evidence for a contribution by purines to the neurogenic response of the guinea-pig urinary bladder. Eur. J. Pharmacol. 87: 415-422.

Wolinsky, E., and P. H. Patterson (1985) Potassium-stimulated purine release by cultured sympathetic neurons. J. Neurosci. 5: 1680-1687.

Yen, S. S., R. L. Klein, S. H. Chen Yen, and A. Thureson-Klein (1976) Norepinephrine: Adenosinetriphosphate ratios in purified adrenergic vesicles. J. Neurobiol. 7: 11-22. 